\title{
Efeito agudo do exercício físico sobre o estado de humor no ambiente de trabalho
}

\author{
Acute effect of a workplace physical activity session on mood
}

Efecto agudo de una sesión de ejercicio físico programado en el trabajo en el estado de ánimo

\section{Rafael Cunba Laux}

Universidade do Oeste de Santa Catarina, Brasil

rafael.laux@unoesc.edu.br

(iD https://orcid.org/0000-0003-2723-3130

\author{
Alexandro Andrade \\ Centro de Ciências da Saúde e do Esporte (CEFID) da \\ Universidade do Estado de Santa Catarina (UDESC), \\ Brasil \\ d2aa@hotmail.com
}

(iD https://orcid.org/0000-0002-6640-9314

\author{
Guilherme Torres Vilarino \\ Centro de Ciências da Saúde e do Esporte (CEFID) da \\ Universidade do Estado de Santa Catarina (UDESC), \\ Brasil \\ guilhermevilarino@hotmail.com \\ (DD https://orcid.org/0000-0002-0879-6723
}

\author{
Sara Teresinha Corazza \\ Centro de Educação Física e Desportos (CEFD) da \\ Universidade Federal de Santa Maria (UFSM), Brasil \\ saratcorazza@gmail.com \\ (D) https://orcid.org/0000-0002-2684-2412
}

\section{Resumo:}

O estudo pretende verificar os efeitos de uma sessão do programa de exercícios físicos no ambiente de trabalho (PEFAT) sobre o estado de humor de servidores públicos de uma universidade. Para tanto, vinte e cinco sujeitos de ambos os sexos e fisicamente inativos, com idade média de 32,16 $\pm 9,64$ anos, foram submetidos a uma sessão de dez minutos de exercício físico no ambiente de trabalho. As avaliações do humor foram realizadas por meio do questionário de humor de Brunel (Brums), em três períodos: i) uma semana antes da intervenção (caracterizada como controle); ii) imediatamente antes a sessão de exercício físico; e iii) logo após a sessão. As análises estatísticas descritiva e inferencial foram realizadas utilizando o software SPSS ${ }^{\circ}$ (versão 21.0 para Windows), com grau de significância de 5\%. Observou-se que não houve mudança entre o estado de humor no período controle e pré intervenção. Na comparação entre pré e pós avaliação observou-se manutenção no aspecto vigor e uma diminuição dos sintomas de tensão $(\mathrm{p}<0,01)$, de raiva $(\mathrm{p}=0,02)$, de fadiga $(\mathrm{p}<0,01)$, e depressão $(\mathrm{p}<0,01)$ e de confusão mental $(\mathrm{p}<0,01)$. Uma sessão do programa de exercícios físicos no ambiente de trabalho produziu efeito agudo positivo no humor de servidores públicos no ambiente de trabalho.

Palavras-chave: Exercício Físico, Estresse Psicológico, Qualidade de Vida.

\section{Abstract:}

The study intends to verify the effects of a workplace physical activity intervention on the humor of university employees in the workplace. Therefore, twenty-five subjects of both sexes and physically inactive, with an average age of $32.16 \pm 9.64$ years, were underwent to a ten-minute session of physical exercise in the workplace. Mood evaluations were performed using Brunel's humor

\section{Recepción: 28 Mayo 2020 | Aprobación: 15 Febrero 2021 | Publicación: 01 Abril 2021}


questionnaire (Brums), in three periods: i) one week before the intervention (characterized as control); Ii) immediately before the physical exercise session; and iii) shortly after the session. Descriptive and inferential statistical analyzes were performed using SPSS $^{\circ}$ software (version 21.0 for Windows), with a significance level of $5 \%$. It was observed that there were no changes between the mood state in the control period and pre intervention. In the comparison between pre and post evaluation, it was observed a maintenance in the aspect of vigor and a decrease in symptoms of tension $(\mathrm{p}<0.01)$, anger $(\mathrm{p}=0.02)$, fatigue $(\mathrm{p}<0.01)$, depression $(\mathrm{p}<0.01)$ and mental confusion $(\mathrm{p}<0.01)$. The workplace physical activity intervention was able to positively influence the mood of the subjects under study.

KEYWORDS: Exercise, Stress Psychological, Quality of Life.

\section{ReSUMEN:}

El estudio pretende verificar los efectos de una sesión del programa de ejercicios físicos en el ambiente laboral (PEFAL) sobre el estado de ánimo de los servidores públicos de una universidad. Por tanto, veinticinco sujetos de ambos sexos y físicamente inactivos, con una edad media de 32,16 \pm 9,64 años, fueron sometidos a una sesión de ejercicio físico de diez minutos en el ámbito laboral. Las evaluaciones del estado de ánimo se realizaron mediante el cuestionario de humor de Brunel (Brums), en tres periodos: i) una semana antes de la intervención (caracterizada como control); ii) inmediatamente antes de la sesión de ejercicio físico; y iii) inmediatamente después de la sesión. Los análisis estadísticos descriptivos e inferenciales se realizaron mediante el software SPSS ${ }^{\circ}$ (versión 21.0 para Windows), con un nivel de significancia del 5\%. Se observó que no hubo cambios entre el estado de ánimo en los períodos de control y preintervención. En la comparación entre pre y post evaluación, se observó mantenimiento en el aspecto vigor y disminución de los síntomas de tensión $(\mathrm{p}<0,01)$, enfado $(\mathrm{p}=0,02)$, fatiga $(\mathrm{p}<0,01)$ y depresión $(\mathrm{p}<0,01)$. ) y confusión mental $(\mathrm{p}<0,01)$. Una sesión del programa de ejercicio físico en el lugar de trabajo produjo un efecto agudo positivo en el estado de ánimo de los servidores públicos en el lugar de trabajo.

Palabras Clave: Ejercicio Físico, Estrés Psicológico, Calidad de Vida.

\section{INTRODUÇÃO}

Em países desenvolvidos grande parte da população trabalha sentada, realizando atividades que requerem pouco gasto de energia (Keadle, Conroy, Dunstan \& Matthews, 2017). Estudos têm demonstrado que quanto maior o tempo que se permanece sentado o risco de morte aumenta, bem como a probabilidade de desenvolver diversas doenças crônicas (Keadle et al., 2017; Van der Ploeg, Chey, Korda, Banks \& Bauman, 2012). Além disso, as mudanças no ambiente de trabalho, que com auxílio dos avanços tecnológicos permitiram maior controle e vigilância sobre os trabalhadores, intensificaram a pressão, o estresse e a ansiedade dos trabalhadores (Santos \& Rocha, 2015), podendo desencadear transtornos psicológicos graves, absenteísmo e redução da capacidade de trabalho (Gerber, Jonsdottir, Lindwall \& Ahlborg, 2014).

Diante dessas novas mudanças no ambiente de trabalho, observa-se que o número de atestados e de afastamentos para cuidar da saúde têm aumentado nos últimos anos. Dados recentes, publicados pelo Departamento Intersindical de Estatística e Estudos Socioeconômicos (DIEESE, 2017), apontam um aumento de $25 \%$ no número de trabalhadores que se afastaram de suas atividades laborais quando comparados aos anos anteriores. Estima-se que doenças musculoesqueléticas sejam responsáveis por cerca de 28\% dos afastamentos enquanto os transtornos mentais por cerca de 20\% (Disler \& Pallant, 2001).

Entre os transtornos mentais, destacam-se os distúrbios do humor, que são responsáveis por ocasionar doenças psíquicas graves, como a depressão (Bartholomew, Morrison \& Ciccolo, 2005), que atinge cerca de 3\% a 5\% da população mundial (Teng, Humes \& Demetrio, 2005; Ferrari et al., 2013), e a ansiedade excessiva, que possui prevalência de 28,8\% (Kessler, Merikangas \& Wang, 2007). No Brasil, a incapacidade por algum distúrbio no humor foi a terceira maior causa para a autorização de benefício auxílio-doença em 2008 (Barbosa-Branco, Bultmann \& Steenstra, 2012). Esses dados têm despertado a atenção de diversos países e têm sido tema de investigação (Silva Junior \& Fischer, 2014; Takala et al., 2017), que buscam estratégias para reduzir esses problemas.

Entre os principais tratamentos para reduzir os distúrbios de humor, observam-se os métodos farmacológicos e não farmacológicos (Bartholomew, Morrison \& Ciccolo, 2005). Entre os tratamentos 
não farmacológicos, destaca-se o exercício físico, que é reconhecido como uma modalidade de tratamento benéfica para melhorar os estados de humor (Bartholomew, Morrison \& Ciccolo, 2005; Meyer, Koltynb, Stegnera, Kimc \& Cook, 2016; Edwards \& Loprinzi, 2016). Assim, a prática regular de exercício físico vem sendo recomendada, pois é considerada um tratamento de fácil implementação e de baixo custo. Além disso, estudos apontam que o exercício físico melhora a saúde mental de diferentes populações, como atletas, trabalhadores e pacientes com doenças reumáticas e com distúrbios psicológicos (Gerber et al., 2014; Andrade, Steffens, Vilarino, Sieczkowaska \& Coimbra, 2017; Brandt, Bevilacqua \& Andrade, 2017).

A importância da investigação dos distúrbios do humor em trabalhadores se justifica pelo fato de que o humor influencia no rendimento dos funcionários (Gerber et al., 2014; Manz, Houghton, Neck, Fugate \&Pearce, 2016). Alguns estudos (Bartholomew et al., 2005; Meyer et al., 2016; Edwards \& Loprinzi, 2016) verificaram que sessões de exercícios aeróbicos com duração de no mínimo 30 minutos alteram de forma benéfica o humor. Outro estudo, investigando o exercício físico no ambiente de trabalho, demonstra que o estado de humor melhora após uma sessão de dez minutos de exercício físico (Stort, Silva Júnior \& Rebustini, 2006). Entretanto, os estudos que investigaram o efeito do exercício no ambiente de trabalho não descrevem detalhadamente o protocolo de exercício (Stort, Silva Júnior \& Rebustini, 2006), dificultando a aplicação prática desse conhecimento, sendo necessário mais estudos sobre o tema.

Desta forma, o objetivo deste estudo foi verificar os efeitos de um programa estruturado de exercícios físicos de curta duração sobre o estado de humor de servidores públicos de uma universidade, no ambiente de trabalho.

\section{MÉTODo}

Este estudo caracterizou-se como experimental, sendo conduzido com os servidores de uma universidade pública de Santa Catarina. O efeito agudo do programa de exercício físico no ambiente de trabalho (PEFAT) foi testado em uma sessão do programa, com duração de dez minutos. As avaliaçes foram realizadas uma semana antes da intervenção (caracterizada como controle), imediatamente antes e após a sessão de exercício físico. Todas as coletas ocorreram no turno vespertino em semana típica de trabalho.

Vinte e cinco sujeitos de ambos os sexos e fisicamente inativos, com idade entre 18 e 50 anos; classificados como sedentários ou insuficientemente ativo A ou B, segundo o IPAQ versão VIII reduzido; e que participaram de todas as etapas propostas, foram recrutados para o estudo (figura 1). Todos os participantes do estudo eram servidores técnico-administrativos de uma universidade federal, na qual desempenhavam atividades laborais sentados em frente ao computador ou com atendimento ao público. 
Figura 1: Fluxograma de seleção dos sujeitos e desenho do estudo
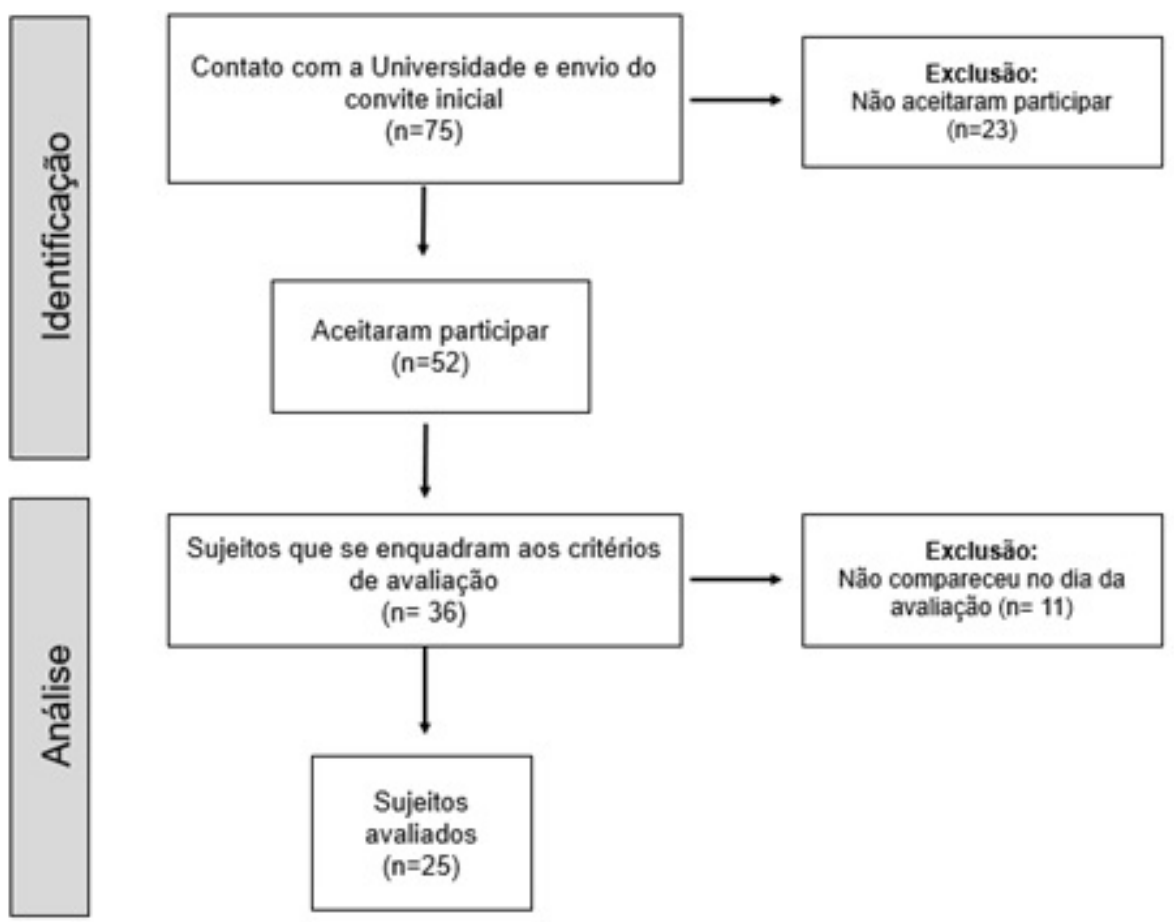

Fonte: os autores.

O projeto foi aprovado pelo Comitê de Ética em Pesquisa da Universidade Federal de Santa Maria pelo CAAE $n^{\circ} 53085216.5 .0000 .5346$, seguindo todos os padrões éticos exigidos pela resolução 466/12 do Conselho Nacional de Saúde.

A caracterização do grupo foi realizada utilizando uma anamnese composta por perguntas como idade e sexo e uma avaliação antropométrica. Para coletar os dados antropométricos de massa corporal e estatura foram utilizados uma balança com resolução digital da marca Filizola (São Paulo, Brasil) e um estadiômetro Cescorf com resolução de $0,1 \mathrm{~cm}$, conforme o protocolo da International Society for the Advancement of Kinanthropometry (ISAK) (Marfell-Jones, Stewart \& De Ridder, 2012) e sua mensuração foi realizada por um avaliador treinado. O cálculo do índice de massa corporal (IMC) seguiu o protocolo da Organização Mundial da Saúde (OMS, 2015).

O nível de prática de exercício físico diário foi verificado por meio do Questionário Internacional de Atividade Física (IPAQ) versão VIII reduzida validado por Matsudo et al. (2001), que, segundo os critérios de classificação, o sujeito é considerado: a) Sedentário, quando não realiza nenhuma atividade física por pelo menos 10 minutos contínuos durante a semana; b) Insuficientemente Ativo A, quando realiza 10 minutos contínuos de atividade física (caminhadas + moderada + vigorosa), com frequência de 5 dias na semana ou duração semanal de 150 minutos; e c) Insuficientemente Ativo B, quando não atinge nenhum dos critérios da recomendação citada nos indivíduos insuficientemente ativos A (Silva et al., 2007).

O estado de humor foi avaliado no período controle, pré e pós intervenção por meio da Escala de Humor de Brunel (BRUMS), validado para população brasileira por Rohlfs et al. (2008). O instrumento é composto por 24 palavras relacionadas aos sentimentos do sujeito naquele momento, no qual o mesmo deve atribuir uma nota de 0 (zero) a 4 (quatro), sendo o 0 quando aquele sentimento não descreve nada para ele e 4 quando ele apresenta extremamente aquele sentimento. A classificação do humor é caracterizada em seis subescalas: raiva, confusão mental, depressão, fadiga, tensão e vigor.

A sessão do PEFAT teve duração de 10 minutos. A aula foi composta por parte inicial ou aquecimento, parte principal e parte final ou relaxamento, baseando-se no protocolo utilizado por Laux, Pagliari, Junior \& Corazza (2016) e na proposta de Laux, Corazza e Andrade (2018), apresentada no quadro 1. 
Quadro 1: Atividades da Sessão do PEFAT

\begin{tabular}{|c|c|c|c|}
\hline & Parte Inicial (4 min) & Parte Principal (4 min) & Parte Final (2 min) \\
\hline ATIVIDADES & $\begin{array}{l}\text { Movimentos de adução } \\
\text { e abdução de braços e } \\
\text { pernas ao ritmo da } \\
\text { música. }\end{array}$ & $\begin{array}{l}\text { Exercícios de } \\
\text { alongamentos } \\
\text { dinâmicos para todos } \\
\text { grupos musculares. }\end{array}$ & $\begin{array}{l}\text { Exercícios } \\
\text { respiração. }\end{array}$ \\
\hline $\begin{array}{l}\text { MATERIAIS } \\
\text { UTILIZADOS }\end{array}$ & $\begin{array}{l}\text { Música com ritmo de } \\
120 \text { a } 140 \mathrm{bpm} \text {. }\end{array}$ & $\begin{array}{l}\text { Música com ritmo de } \\
100 \text { a } 120 \mathrm{bpm} \text {. }\end{array}$ & Música relaxante. \\
\hline
\end{tabular}

Fonte: adaptado de Laux et al. (2016).

A intensidade dos exercícios foi controlada utilizando a escala de percepção subjetiva do esforço (Borg, 2000), de maneira a manter uma intensidade entre um pouco intensa e intensa (13-15 pontos na escala) durante a sessão.

Os dados foram analisados de maneira descritiva (média e desvio padrão) e inferencial. A normalidade dos dados foi verificada com o teste Shapiro-Wilk, que identificou uma distribuição não paramétrica para todos os aspectos negativos do estado humor e paramétrico para o vigor. Para comparação entre os três períodos, utilizou-se o teste inferencial Friedman para os aspectos negativos do estado de humor, Anova de medidas repetidas para o vigor, seguido do teste de Bonferroni como Post Hoc. Na comparação entre pré e pós teste foi utilizado o teste Wilcoxon nos aspectos negativos do estado de humor e test $\mathrm{T}$ pareado para o vigor. $\mathrm{O}$ grau de significância adotado foi de 5\% e o software estatístico utilizado foi o SPSS (versão 21.0 para Windows).

\section{Resultados}

Participaram do estudo vinte e cinco sujeitos, 15 mulheres e 10 homens, fisicamente inativos, com idade

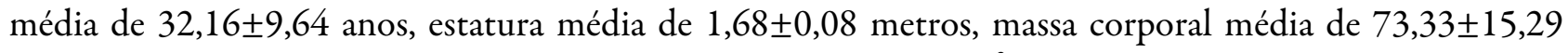
quilogramas e ndice de Massa Corporal (IMC) de $25,86 \pm 4,52 \mathrm{~kg} / \mathrm{m}^{2}$.

A Tabela 1 apresenta os valores médios da escala de humor na avaliação controle, pré e pós intervenção. Observou-se que não houve diferença nos diferentes aspectos do humor entre o momento controle e pré intervenção. Porém, verificou-se uma diminuição no momento pós-intervenção na tensão $(\mathrm{p}<0,01)$, na raiva $(\mathrm{p}<0,01)$, na fadiga $(\mathrm{p}<0,01)$ e na confusão mental $(\mathrm{p}<0,01)$ dos participantes da intervenção. $\mathrm{O}$ vigor e a depressão não tiveram alteração com a intervenção proposta, mesmo o teste estatístico apresentando diferença, o post hoc não foi capaz de identificar onde houve a mudança, portanto, optou-se por analisar também o modelo somente com o pré e o pós intervenção (Figura 2). 
Tabela 1: Comparação do estado de humor no período de controle, pré e pós intervenção

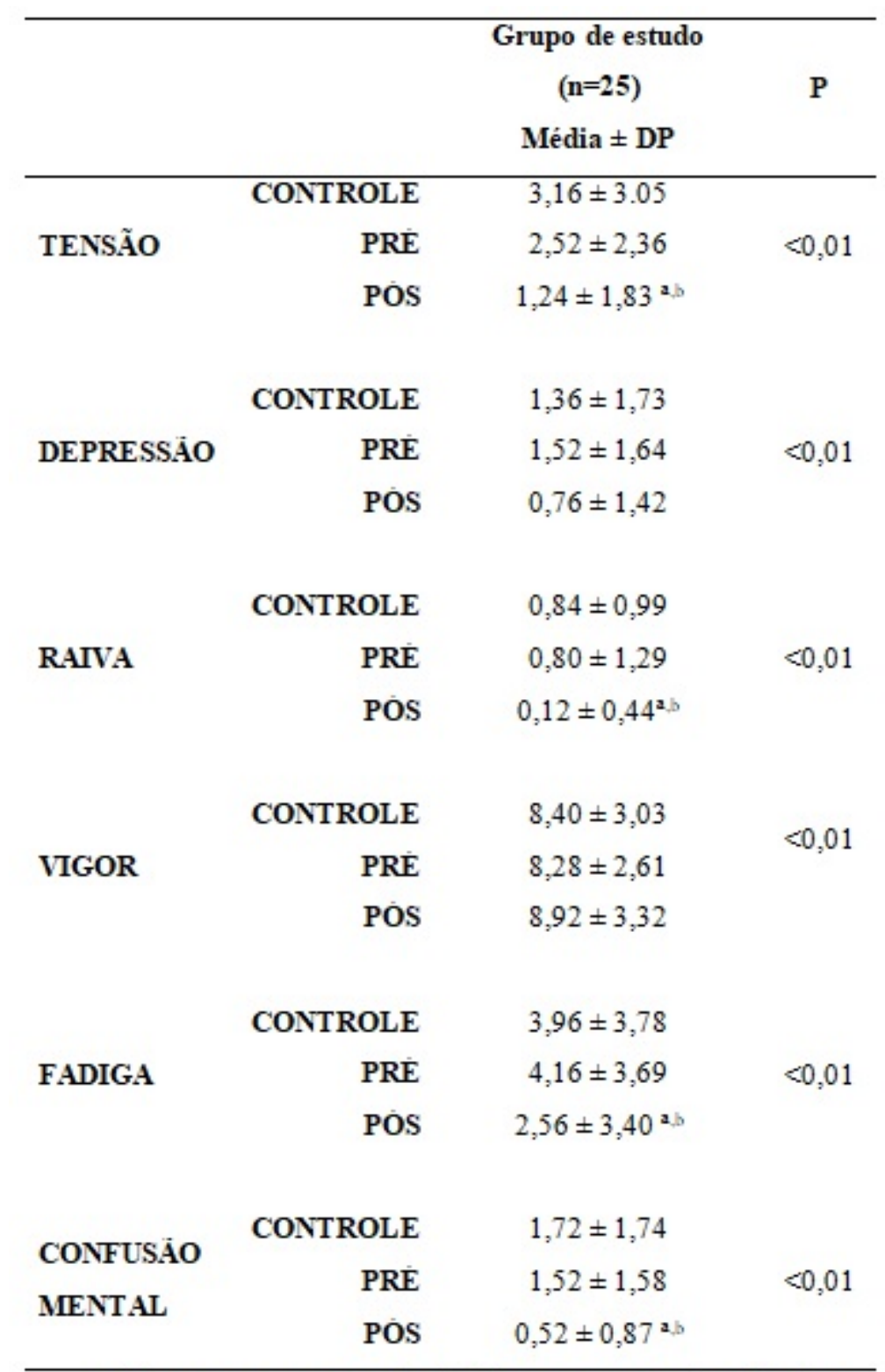

' diferença com o controle, ${ }^{b}$ diferença com a pré-avaliação.

Fonte: os autores.

$\mathrm{Na}$ Figura 2 são apresentados os estados de humor pré e pós intervenção, nos quais pode-se verificar uma diminuição nos sintomas de tensão $(\mathrm{p}<0,01)$, de depressão $(\mathrm{p}<0,01)$, de raiva $(\mathrm{p}=0,02)$, de fadiga $(\mathrm{p}<0,01)$ e de confusão mental $(\mathrm{p}<0,01)$. $\mathrm{O}$ vigor não teve alteração com a intervenção proposta. Os resultados apresentam o clássico perfil iceberg, com baixos escores nos aspectos negativos do humor e altos no vigor. 
Figura 2: Perfil Iceberg do estado de humor pré e após uma sessão de exercício

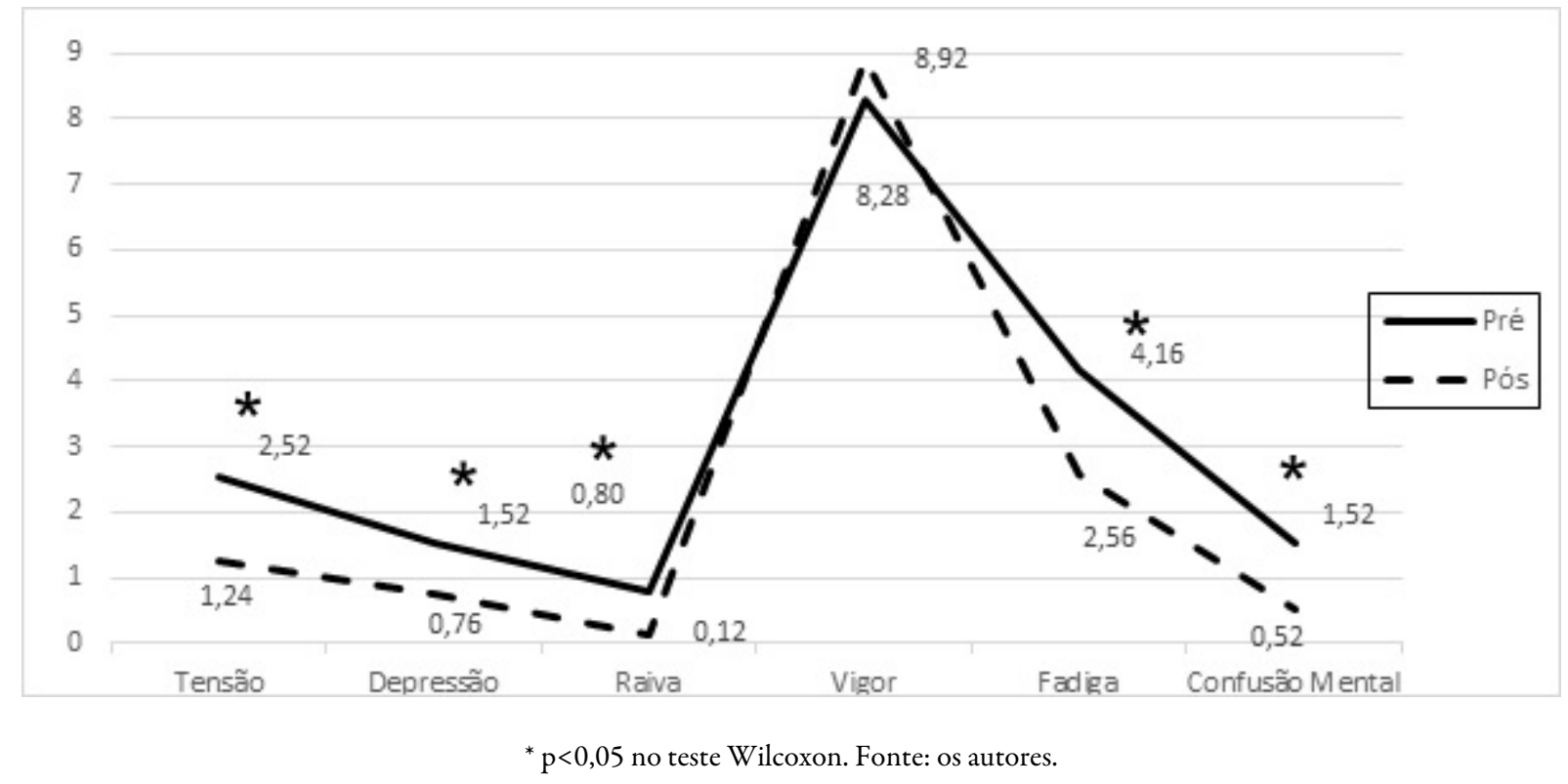

\section{Discussão}

Durante a verificação do efeito agudo de uma sessão de exercício físico sobre o humor de servidores públicos de uma universidade, no ambiente de trabalho, observou-se a diminuição de todos os aspectos negativos do humor na comparação entre pré e pós avaliação. Bartholomew, Morrison e Ciccolo (2005) destacam a importância de intervenções que diminuam esses elementos, principalmente para sujeitos com níveis mais elevados de depressão, enfatizando que o próprio ambiente de trabalho pode ser o responsável por desencadear esses distúrbios no humor (Manz et al., 2016).

A melhora nos aspectos negativos do humor influencia no desempenho do trabalhador, uma vez que a tensão, a raiva, a fadiga e a confusão mental tem correlação negativa com o desempenho psicomotor, o tempo de reação e a eficácia mental (Bolmont, Thullier \& Abraini, 2000), capacidades necessárias para tomadas de decisões. Esses elementos são fundamentais no trabalho, o tempo de reação, por exemplo, pode evitar acidentes neste ambiente, pois o sujeito com maior performance nessa variável responde mais rápido a situações de risco (Wilkerson, Simpson \& Clark, 2017).

O estudo de Stort, Silva Júnior e Rebustini (2006) que avaliou o estado de humor pré e pós intervenção de uma sessão com exercícios compensatórios e de alongamentos em funcionários de uma empresa multinacional de São Paulo, verificou a melhora em todas as dimensões do humor, dados similares aos do presente estudo. Porém, o estudo de Freitas-Swerts e Robazzi (2014) não verificou melhora no estresse ocupacional com sessões de 15 minutos de ginástica laboral compensatória.

Os participantes do presente estudo apresentaram um perfil de humor denominado de perfil iceberg, no qual constatou-se escores elevados do aspecto vigor e baixos dos demais fatores tidos como elementos negativos do humor. Esse perfil é caracterizado como um perfil de humor ideal para que um sujeito atinja o seu melhor desempenho em tarefas físicas, psicológicas e emocionais (Prado et al., 2020). O fator vigor, aspecto positivo do estado de humor, representa um estado de energia, vitalidade e vigor físico (Bevilacqua, Viana, Gutierres Filho, Borges \& Brandt, 2019).

O fato desses trabalhadores terem apresentado esse perfil pode ser em decorrência da intensidade do exercício as quais eles foram submetidos. Sabe-se que intensidades moderadas proporcionam melhora desse estado de humor, enquanto altas intensidades estão associadas à piora dele (Silva et al., 2014). Além do benefício psicológico do exercício estar atrelado à individualidade do praticante, às características do 
exercício, como tipo, intensidade, volume, e ao ambiente em que se pratica (Silva \& Ferreira, 2011). Todos esses fatores podem ter influenciado os trabalhadores a, mesmo após a prática de exercício físico, apresentarem um perfil de humor positivo.

Ademais, deve-se destacar a pequena quantidade de estudos com efeito agudo ou crônico do exercício físico no ambiente de trabalho. Entretanto, tem-se visto um aumento no número de estudos mostrando os benefícios do exercício sobre o humor, principalmente os exercícios aeróbios (Bartholomew, Morrison \& Ciccolo, 2005; Meyer et al., 2016). Outro estudo indica que o comportamento sedentário é causador do aumento da depressão e mudanças negativas no estado de humor (Edwards \& Loprinzi, 2016).

\section{CONCLUSÃo}

Em conclusão, a partir dos resultados do presente estudo verificou-se uma diminuição dos aspectos negativos do humor e a manutenção do alto nível de vigor dos sujeitos submetidos ao programa estruturado de exercícios físicos de curta duração no ambiente de trabalho. Sugere-se novos estudos de efeito agudo e crônico, comparando diversos métodos de exercício físico no ambiente de trabalho, para a verificação de qual é o mais eficaz na melhora do estado de humor.

No decorrer da investigação, algumas limitações foram encontradas, entre elas o pequeno número de sujeitos e tempos reduzidos para aplicação da intervenção. Entretanto, destaca-se a rigorosa seleção dos participantes, os cuidados na escolha da semana da intervenção e a aplicação no próprio ambiente de trabalho como pontos que fortalecem os achados do presente estudo e sua aplicação no cotidiano das instituiçóes para melhora do humor.

\section{Conflito DE INTERESSES}

Não houve qualquer conflito de interesse na realização desse trabalho.

\section{REFERÊNCIAS}

Andrade, A., Steffens, R. D. A. K., Vilarino, G. T., Sieczkowska, S. M., \& Coimbra, D. R. (2017). Does volume of physical exercise have an effect on depression in patients with fibromyalgia?. Journal of affective disorders, 208, 214-217.

Barbosa-Branco, A., Bültmann, U., \& Steenstra, I. (2012). Sickness benefit claims due to mental disorders in Brazil: associations in a population-based study. Cadernos de saude publica, 28, 1854-1866.

Bartholomew, J. B., Morrison, D., \& Ciccolo, J. T. (2005). Effects of acute exercise on mood and well-being in patients with major depressive disorder. Medicine \& science in sports \& exercise, 37(12), 2032-2037.

Bevilacqua, G. G., Viana, M. da S., Gutierres Filho, P. J. B., Borges, V. da S., \& Brandt, R. (2019). Estados de Humor e Resultado Esportivo de Uma Equipe ao Longo da Segunda Fase da Liga Nacional de Futsal. Psicologia: Teoria e Pesquisa, 35.

Bolmont, B., Thullier, F., \& Abraini, J. H. (2000). Relationships between mood states and performances in reaction time, psychomotor ability, and mental efficiency during a 31-day gradual decompression in a hypobaric chamber from sea level to $8848 \mathrm{~m}$ equivalent altitude. Physiology \& behavior, 71(5), 469-476.

Borg, G. (2000). Escalas de Borg para a dor eo esforço: percebido. Manole.

Brandt, R., Bevilacqua, G. G., \& Andrade, A. (2017). Perceived sleep quality, mood states, and their relationship with performance among Brazilian elite athletes during a competitive period. Journal of strength and conditioning research, 31(4), 1033-1039. 
Departamento Intersindical de Estatística e Estudos Socioeconômicos (2017). Anuário do sistema público de emprego, trabalho e renda 2016. São Paulo: Ministério do trabalho.

Disler PB \& Pallant JF (2001). Vocational rehabilitation. BMJ, 323(7305), 121-123.

Edwards, M. K., \& Loprinzi, P. D. (2016, August). Effects of a sedentary behavior-inducing randomized controlled intervention on depression and mood profile in active young adults. In Mayo Clinic Proceedings (Vol. 91, No. 8, pp. 984-998). Elsevier.

Ferrari, A. J., Somerville, A. J., Baxter, A. J., Norman, R., Patten, S. B., Vos, T., \& Whiteford, H. A. (2013). Global variation in the prevalence and incidence of major depressive disorder: a systematic review of the epidemiological literature. Psychological medicine, 43(3), 471-481.

Freitas, F. C. T., Swerts, O. S. D., \& Robazzi, M. L. C. C. (2014). Efeitos da ginástica laboral compensatória na redução do estresse ocupacional e dor muscular. Revista Latino-Americana Enfermagem, 22(4), 629-639.

Gerber, M., Jonsdottir, I. H., Lindwall, M., \& Ahlborg Jr, G. (2014). Physical activity in employees with differing occupational stress and mental health profiles: A latent profile analysis. Psychology of Sport and Exercise, 15(6), 649-658.

Keadle, S. K., Conroy, D. E., Buman, M. P., Dunstan, D. W., \& Matthews, C. E. (2017). Targeting reductions in sitting time to increase physical activity and improve health. Medicine and science in sports and exercise, 49(8), 1572.

Kessler, R. C., Merikangas, K. R., \& Wang, P. S. (2007). Prevalence, comorbidity, and service utilization for mood disorders in the United States at the beginning of the twenty-first century. Annu. Rev. Clin. Psychol., 3, 137-158.

Laux, R. C., Corazza, S. T., \& Andrade, A. (2018). Workplace physical activity program: an intervention proposal. Revista Brasileira de Medicina do Esporte, 24(3), 238-242.

Laux, R. C., Pagliari, P., Junior, J. V. E., \& Corazza, S. T. (2016). Programa de Ginástica Laboral e a Redução de Atestados Médicos. Revista Ciência \& Trabalho.

Manz, C. C., Houghton, J. D., Neck, C. P., Fugate, M., \& Pearce, C. (2016). Whistle while you work: Toward a model of emotional self-leadership. Journal of Leadership \& Organizational Studies, 23(4), 374-386.

Marfell-Jones, M. J., Stewart, A. D., \& De Ridder, J. H. (2012). International standards for anthropometric assessment.

Matsudo, S., Araújo, T., Matsudo, V., Andrade, D., Andrade, E., Oliveira, L. C., \& Braggion, G. (2001). Questionário internacional de atividade física (IPAQ): estupo de validade e reprodutibilidade no Brasil. Revista Brasileira de Atividade Física \& Saúde, 6(2), 5-18.

Meyer, J. D., Koltyn, K. F., Stegner, A. J., Kim, J. S., \& Cook, D. B. (2016). Relationships between serum BDNF and the antidepressant effect of acute exercise in depressed women. Psychoneuroendocrinology, 74, 286-294.

Prado, V. L. de O., Dorneles, S. P., Oliveira, F. A. de, Garcia, R. L. da S., Machado, A. A., \& Tertuliano, I. W. (2020). Estado de Humor de atletas de categoria de base da modalidade Basquetebol. Pensar a Prática, 23.

Rohlfs, I. C. P. D. M., Rotta, T. M., Luft, C. D. B., Andrade, A., Krebs, R. J., \& Carvalho, T. D. (2008). A Escala de Humor de Brunel (Brums): instrumento para detecção precoce da síndrome do excesso de treinamento. Revista Brasileira de Medicina do Esporte, 14(3), 176-181.

Santos, F. P., \& da Rocha, M. A. H. (2015). Depressão ocupacional: impacto na saúde mental do colaborador. Brazilian Journal of Health, 3(2).

Silva Junior, J. S. D., \& Fischer, F. M. (2014). Disability due to mental illness: social security benefits in Brazil 2008-2011. Revista de saude publica, 48, 186-190.

Silva, G. S. F., Bergamaschine, R., Rosa, M., Melo, C., Miranda, R., \& Bara Filho, M. (2007). Evaluation of the physical activity level of undergraduation students of health/biology fields. Rev Bras Med Esporte, 13(1), 39-42.

Silva, P. S. B. da, \& Ferreira, C. E. S. (2011). Exercício físico e humor: uma revisão acerca do tema. Educação Física em Revista, 5(3), Article 3. https://portalrevistas.ucb.br/index.php/efr/article/view/2811

Silva, V. B., Bigliassi, M., Kanthack, T. F. D., Souza, S. R., Lanaro Filho, P., \& Altimari, L. R. (2014). Influência de Diferentes Protocolos de Exercício Cíclico Sobre o Estado de Humor. Revista Brasileira de Ciência e Movimento, 22(4), 146-155. https://doi.org/10.18511/0103-1716/rbcm.v22n4p146-155 
Stort, R., Silva Junior, F. P., \& Rebustini, F. (2006). Os efeitos da atividade física nos estados de humor no ambiente de trabalho. Revista Brasileira de Educação Fisica, Esporte, Lazer e Dança, 1(1), 26-33

Takala, J., Hämäläinen, P., Nenonen, N., Takahashi, K., Chimed-Ochir, O., \& Rantanen, J. (2017). Comparative analysis of the burden of injury and illness at work in selected countries and regions. Cent. Eur. J. Occup. Environ. Med, 23, 6-31.

Teng, C. T., Humes, E. D. C., \& Demetrio, F. N. (2005). Depressão e comorbidades clínicas. Archives of Clinical Psychiatry (São Paulo), 32(3), 149-159.

Van der Ploeg, H. P., Chey, T., Korda, R. J., Banks, E., \& Bauman, A. (2012). Sitting time and all-cause mortality risk in 222497 Australian adults. Archives of internal medicine, 172(6), 494-500.

Wilkerson, G. B., Simpson, K. A., \& Clark, R. A. (2017). Assessment and training of visuomotor reaction time for football injury prevention. Journal of sport rehabilitation, 26(1), 26-34.

World Health Organization. (2015). Obesity and overweight. Fact sheet No 311. 2015. WHO Media centre URL: http://www.who.int/mediacentre/factsheets/fs311/en/ [accessed 2015-12-24][WebCite Cache ID $6 \mathrm{e} 0 \mathrm{qY} c 8 \mathrm{mX}]$. 